\title{
One day is better than four days of ejaculatory abstinence for sperm function
}

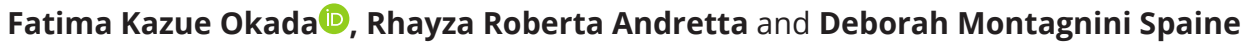 \\ Human Reproduction Section, Division of Urology, Department of Surgery, Federal University of São Paulo, R. Borges Lagoa, São Paulo, São Paulo, Brazil \\ Correspondence should be addressed to F K Okada: fatimaokada@gmail.com
}

\begin{abstract}
According to the World Health Organization guidelines, ejaculatory abstinence (EA) of 2-7 days is recommended for semen analysis. This study aimed to determine how seminal quality may be affected by two EA periods from the same man. Seminal samples from 65 men were evaluated by conventional semen analysis and qualitative characteristics after 1 and 4 days of EA (two samples/man). The semen was qualitatively analyzed by examining oxidative activity (intracellular and seminal plasma), sperm function (acrosome integrity, mitochondrial activity, and nuclear DNA integrity), and epididymal function. As expected, samples collected after 1 day of EA showed a decrease in volume and sperm total number compared to samples collected after 4 days of EA. The sperm motility of the samples collected after 1 day of EA was better compared to samples collected after 4 days of EA. Oxidative activity measured was lower after 1 day of EA compared with those measured after 4 days of EA. With regards to sperm function, samples collected after 1 day of EA showed an increase in acrosome integrity, mitochondrial activity, and nuclear DNA integrity compared with samples collected after 4 days of EA. Epididymal function showed no difference between the two-time points. Although samples collected after 4 days of EA showed better results for sperm quantity, samples collected after 1 day of EA showed better qualitative results, including motility, oxidative activity, and sperm function. Thus, it can be concluded that sperm storage at the epididymal tail may make spermatozoa more susceptible to oxidative damage.
\end{abstract}

\section{Lay summary}

According to the World Health Organization guidelines, stopping ejaculation for 2 to 7 days is recommended before sperm collection for semen analysis. However, the evidence that supports these recommendations is limited. Our study aimed to compare how sperm quality was affected in samples collected after stopping ejaculation for 1 day and 4 days (two samples per man) in a total of 65 men. Although sample collection after stopping ejaculation for 4 days showed better semen quantity (volume and sperm concentration), sample collection after stopping ejaculation for 1 day showed better sperm motility and function. If not ejaculated, sperm are stored in the epididymis tail located in the scrotum beside the testicles and our study suggests that longer sperm storage may damage sperm quality. The results from this study may be used to inform guidance for sperm collection for use in assisted reproduction techniques, and lead to an improvement in both fertilization and implantation rates.

Key Words: • ejaculatory abstinence $\quad$ sperm $\quad$ oxidative stress $\quad$ DNA fragmentation $\quad$ superoxide anion Reproduction and Fertility (2020) 1 1-11

(c) 2020 The authors Published by Bioscientifica Ltd

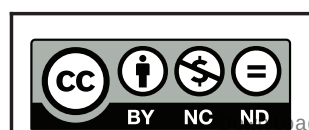

This work is licensed under a Creative Commons Attribution-NonCommercial-NoDerivatives 4.0 International License. 


\section{Introduction}

Assisted reproduction is a technique used by couples with conjugal infertility, which affects around 15\% of couples of reproductive age. The male partner alone accounts for $50 \%$ of these cases (WHO 2010). For this reason, it is important to obtain more information about sperm quality. According to the World Health Organization (WHO) guidelines, ejaculatory abstinence (EA) of 2-7 days is recommended for semen analysis (WHO 2010); nevertheless, the evidence that supports these recommendations is limited, arbitrary, and requires clarification (Ayad et al. 2018).

Some studies have verified the effects of the EA period on sperm quality and seminal parameters (Agarwal et al. 2016, Ayad et al. 2018, Borges et al. 2019); however, few studies have reported the effects of the EA period on sperm function, such as oxidative activity (Marshburn et al. 2014), nuclear DNA integrity (Agarwal et al. 2016, Uppangala et al. 2016, Borges et al. 2019), mitochondrial activity (Shen et al. 2019), and acrosome integrity (Levitas et al. 2006, Shen et al. 2019).

Mayorga-Torres et al. (2015) have shown that a longer EA is associated with increases in semen volume and sperm count. Sperm motility, viability (De Jonge et al. 2004, Rivaroli 2009, Mayorga-Torres et al. 2015, Agarwal et al. 2016), morphology (Carlsen et al. 2004, Sobreiro et al. 2005, Acuña et al. 2013, Welliver et al. 2016), and nuclear DNA integrity (Carlsen et al. 2004, De Jonge et al. 2004, Mayorga-Torres et al. 2016, Welliver et al. 2016) data are inconclusive; however, there are some publications that semen parameters are improved with a shorter EA period (De Jonge et al. 2004, Hanson et al. 2018, Borges et al. 2019).

Considering all this information, we hypothesized that reactive oxidative species (ROS) may act on sperm during the EA period and lead to decreased seminal quality.

While the WHO guidelines (WHO 2010) recommend 2-7 days of EA for seminal analysis, since 2010, many other assays have been developed in order to evaluate seminal samples in more depth. Thus, the aim of this study was to understand which sperm functions may be affected by the EA period when analyzing semen samples from the same man. To this end, we evaluated conventional parameters and qualitative characteristics on semen, such as seminal oxidative activity (intracellular and seminal plasma), sperm function (acrosome integrity, mitochondrial activity, and DNA integrity), and epididymal function.

\section{Materials and methods}

Samples were calculated using a GPower 3.1.10 software (for 0.05 alpha level and 0.90 power). The effect size was calculated using sperm DNA integrity data from other published literature (Pons et al. 2013, SánchezMartín et al. 2013), which showed that a total sample size of 44 men was required.

To avoid errors during the study performance, several precautions were taken: only one person performed samples per evaluation method; samples were analyzed blindly during the study; and all members of this research were trained in a responsible evaluation method. All recommendations on quality control were followed in accordance with the WHO guidelines (WHO 2010); moreover, all utilized equipment were routinely calibrated.

\section{Men and guidance on the study}

Participants were recruited from the general population through doctors during the first medical consultation or dissemination on social media and pamphlets attached in places with a large circulation of people - about the possibility to participate in this study. Those who agreed, signed a written informed consent form approved by the local Institutional Review Board. The participants were instructed in detail about this study, and mainly about the importance of EA in this research.

After scheduling the seminal collections, the volunteers received an e-mail reminding them of the importance of maintaining EA for this study. Besides, 1 week before the first scheduled collection, they received a phone call with the same recommendations.

The men collected two seminal samples (after 1 and 4 days of EA) at the Andrology Laboratory of the São Paulo Federal University, in the same week. The participants were informed about the best time to perform masturbation 'at home' before the first scheduled laboratory collection. After the first collection, they were reminded about the importance of maintaining EA until the next scheduled laboratory collection.

\section{Seminal samples and processing}

For this study, 65 men (research volunteers) were recruited between June 2014 and April 2017. All men included in the study provided two seminal samples: 1 day (about $24 \mathrm{~h}$ ) and 4 days (about $96 \mathrm{~h}$ ) after EA, collected at the Andrology Laboratory. Seminal samples (after 1 and

This work is licensed under a Creative Commons Attribution-NonCommercial-NoDerivatives 4.0 International License. International License.
aded from Bioscientifica.com at 04/26/2023 01:50:43PM 
Table 1 Inclusion and exclusion criteria for seminal and clinical parameters.

Inclusion criteria
Age between 20 and 45 years
Exclusion criteria
Azoospermia*
Sperm concentration $<5 \times 106 / \mathrm{mL}$
Seminal volume $<1 \mathrm{~mL}$
Body mass index $>35 \mathrm{~kg} / \mathrm{m}^{2}$
Leukocytostermia
History of cryptorchidism\#
History of urogenital surgical interventions ${ }^{*}$
History of systemic diseases
History of smoking or excessive alcohol or drug consumption
Episodes of fever in the 90 days before the seminal analysis

*Azoospermia (no spermatozoa in the ejaculate after centrifugation); \#Failure to descend of one or both testes into the scrotum; 'For example, prostatectomy, vasectomy, vasovasostomy, testicular torsion repair, among others; *For example, cancer, diabetes mellitus, endocrinopathies, among others and their treatments that lead to testicular alterations.

4 days of EA) were collected in the same week. Table 1 presents the inclusion and exclusion criteria for seminal and clinical parameters.

After semen liquefaction, volume, motility, $\mathrm{pH}$, sperm concentration, sperm normal morphology, round cell concentration, and neutrophil concentration were measured following WHO standard procedures (WHO 2010), and the semen analysis checklist by Björndahl et al. (2016) was performed (Supplementary Table, see section on supplementary materials given at the end of this article).
The remaining semen volume was divided into three aliquots: (i) analysis of intracellular oxidative activity (superoxide anion) and sperm function (acrosome integrity, mitochondrial activity, nuclear DNA integrity); (ii) another aliquot was centrifuged at $1000 \boldsymbol{g}$ for $10 \mathrm{~min}$ at room temperature; and (iii) the last aliquot was centrifuged at $16,000 \mathrm{~g}$ for $1 \mathrm{~h}$ at $4^{\circ} \mathrm{C}$ to remove of the cellular debris. The last two aliquots of the seminal plasma were stored at $-20^{\circ} \mathrm{C}$ for further analysis of epidydimal function and seminal plasma oxidative activity, respectively. All reagents used in this study were purchased from SigmaAldrich, unless otherwise described.

\section{Evaluation of sperm intracellular oxidative activity}

The sperm intracellular oxidative activity was evaluated by measuring intracellular superoxide anion levels using a dihydroethidium probe (DHE - D11347 - Thermo Fisher Scientific) (Mahfouz et al. 2010, Adami et al. 2018). The samples were incubated in a proportion of 1:1 (sample volume: DHE solution volume), in which the DHE solution contained $2 \mu \mathrm{mol} / \mathrm{L}$ DHE and $16 \mu \mathrm{mol} / \mathrm{L}$ Hoechst H33342 in PBS (137 mmol/L NaCl, $2.7 \mathrm{mmol} / \mathrm{L} \mathrm{KCl}$, $10 \mathrm{mmol} / \mathrm{L} \mathrm{Na}_{2} \mathrm{HPO}_{4}$, and $\left.1.8 \mathrm{mmol} / \mathrm{L} \mathrm{KH}_{2} \mathrm{PO}_{4}\right)$. Then, the samples were incubated in the dark at room temperature for $15 \mathrm{~min}$. After that, the sample was pipetted onto two microscope slides and covered with coverslip. A total of 100 sperm per replicate (200 per seminal sample) was counted using an Olympus BX51 epifluorescence
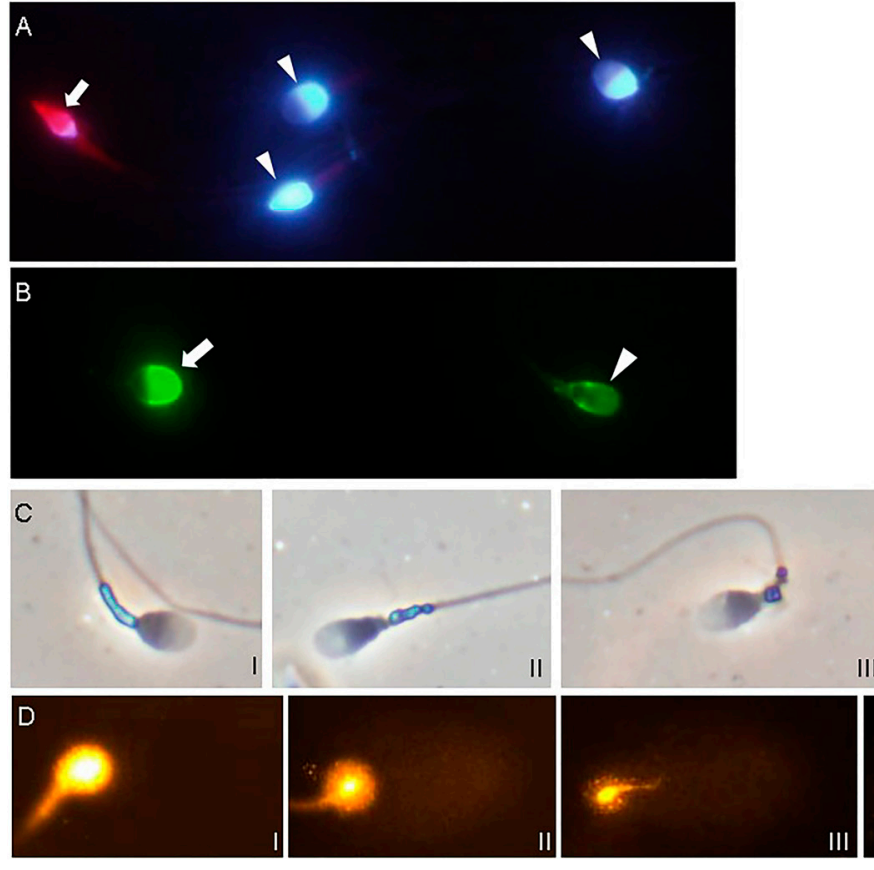

https://raf.bioscientifica.com https://doi.org/10.1530/RAF-20-0018 (c) 2020 The authors Published by Bioscientifica Ltd

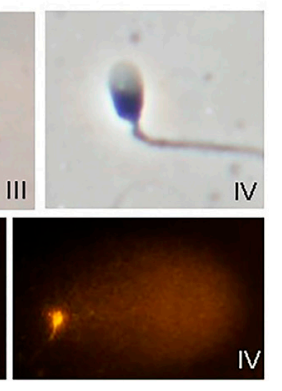

Figure 1 Photomicrographs of human sperm after the following evaluations were performed. (A) Sperm intracellular oxidative activity: DHE-positive (arrow) and DHE-negative (head arrow) sperm are shown. (B) Acrosome integrity: intact acrosome (arrow) and non-intact acrosome (head arrow) sperm were observed. (C) Mitochondrial activity: sperm are shown according to the classification order: DAB class I - $100 \%$ staining (I), DAB class II - more than $50 \%$ stained (II), DAB III - less than $50 \%$ stained (III), DAB IV - without staining (IV). (D) Nuclear DNA integrity: sperm are shown according to the classification order: Comet grade I - observed nucleus and no DNA migration (I), Comet grade II - observed nucleus and little DNA migration (II), Comet grade III - observed nucleus and intense DNA migration (III), Comet grade IV - no observed nucleus and intense DNA migration (IV). 
microscope equipped with a wide-pass filter to detect DHE (518-606 nm) and Hoechst (350-461 nm) under 1000x magnification. Subsequently, the sperm was classified as DHE-positive or DHE-negative (Fig. 1A). The percentage of the DHE-positive sperm was then calculated for the sample.

\section{Evaluation of seminal plasma oxidative activity}

The seminal plasma oxidative activity was evaluated by measuring the level of malondialdehyde (MDA), which is a product of lipid peroxidation. One molecule of MDA reacts with two molecules of thiobarbituric acid (TBA) at high temperatures and low $\mathrm{pH}$, resulting in a pink complex, as described by Ohkawa et al. (Ohkawa et al. 1979, Intasqui et al. 2015). The seminal plasma aliquot, which was centrifuged at $16,000 \boldsymbol{g}$ and pre-frozen, was thawed and added to a solution containing $8.1 \%$ sodium dodecyl sulfate (w/v), 0.8\% TBA (w/v), and 20\% acetic acid (v/v). The microtubes with sample were closed and incubated in a water bath at $100^{\circ} \mathrm{C}$ for $1 \mathrm{~h}$. After that, they cooled in ice for $5 \mathrm{~min}$ to stop the reaction and the volume was checked. If any changes were observed, the microtube was excluded and the analysis repeated. Then, $250 \mu \mathrm{L}$ of N-butanol was added to each sample to separate the thiobarbituric acid reactive substances (TBARS). The sample was later homgenized in a vortex for $1 \mathrm{~min}$. It was centrifuged at $16,000 \mathrm{~g}$ at $15^{\circ} \mathrm{C}$ for $15 \mathrm{~min}$ and $100 \mu \mathrm{L}$ of the top layer was transferred to a 96-well microplate in duplicate. A standard curve was prepared using a known MDA concentration $(0-50 \mathrm{ng} / \mathrm{mL})$, and underwent the same protocol as the seminal samples. It was added to a 96-well microplate in duplicate. The absorbance was read by spectrophotometry (ELx 800 Absorbance Microplate Reade, Biotek) at a wavelength of $540 \mathrm{~nm}$. The lipid peroxidation level was determined as TBARS/mL of seminal plasma.

\section{Evaluation of acrosome integrity}

The acrosomal integrity was evaluated using a peanut agglutinin conjugated to a fluorescein isothiocyanate probe (PNA-FITC; Sigma, L7381) that binds to the outer acrosome membrane (Blumer et al. 2012, Intasqui et al. 2016). Two $15 \mu \mathrm{L}$ smears were placed on microscope slides and air dried. The slides were tehn fixed in methanol (Merck) for $15 \mathrm{~min}$ and air dried again. The sperm was stained with $60 \mu \mathrm{g} / \mathrm{mL}$ of PNA-FITC in PBS $(137 \mathrm{mmol} / \mathrm{L}$ $\mathrm{NaCl}, 2.7 \mathrm{mmol} / \mathrm{L} \mathrm{KCl}, 10 \mathrm{mmol} / \mathrm{L} \mathrm{Na}_{2} \mathrm{HPO}_{4}$, and $1.8 \mathrm{mmol} / \mathrm{L} \mathrm{KH}_{2} \mathrm{PO}_{4}$ ) for $30 \mathrm{~min}$ in the dark and washed with milli-Q water to remove background staining. A total of 200 sperm (100 sperm per slide) was analyzed using an Olympus BX-51 epifluorescence microscope equipped with an FITC filter under 1000× magnification. The sperm was classified as in intact acrosome or non-intact acrosome (Fig. 1B). The percentage of sperm with intact acrosome for sample was then calculated.

\section{Evaluation of mitochondrial activity}

The mitochondrial activity was evaluated using the 3,3'-diaminobenzidine chromogen (DAB), which is oxidized by the cytochrome c complex, in a chain reaction in which the reagent is polymerized and deposited in the sperm mid-piece (Hrudka 1987, Intasqui et al. 2016). The samples were incubated in a proportion of 1:1 to $1: 3$ (sample volume:DAB solution volume), in which DAB solution contained $1 \mathrm{mg} / \mathrm{mL}$. The samples were incubated in a water bath at $37^{\circ} \mathrm{C}$ for $1 \mathrm{~h}$ in the dark. Then, two $15 \mu \mathrm{L}$ smears were placed on microscope slides and air dried. The slides were fixed in $10 \%$ formaldehyde for 10 min and air dried again. A total of 200 sperm (100 sperm per slide) was analyzed using an Olympus BX-51 phase contrast optical microscope under 1000x magnification. The sperm was scored according to the level of mid-piece staining and classified into DAB class I (100\% stained), DAB class II (more than 50\% stained), DAB class III (less than $50 \%$ stained), or DAB class IV (without staining) (Fig. 1C). The percentage of each class of sample was then calculated.

\section{Evaluation of nuclear DNA integrity}

The nuclear DNA fragmentation was evaluated using the alkaline comet assay (Comet) (Blumer et al. 2008, Intasqui et al. 2016). The samples were diluted in $0.75 \%$ low melting point agarose in Tris-borate-EDTA buffer (TBE, $0.089 \mathrm{~mol} / \mathrm{L}$ Tris, $0.089 \mathrm{~mol} / \mathrm{L}$ borate, and 0.002 $\mathrm{mol} / \mathrm{L}$ EDTA) at $37^{\circ} \mathrm{C}$ to a concentration of $1 \times 10^{6}$ cells/ mL. $100 \mu \mathrm{L}$ of diluted sperm solution was placed on each slide already pre-covered with $1 \mathrm{~mL}$ of $1 \%$ normal melting point agarose in TBE. In all subsequent steps, the slides were protected from light to prevent artificial DNA damage. The slides were covered with a coverslip and kept at $4^{\circ} \mathrm{C}$ for $10 \mathrm{~min}$ to solidify. The coverslips were then removed and $300 \mu \mathrm{L}$ of $0.75 \%$ low melting point agarose in TBE was added. The slides were covered with a coverslip again and kept at $4^{\circ} \mathrm{C}$ for $10 \mathrm{~min}$. After that, the coverslips were removed and $1 \mathrm{~mL}$ of cold lysis buffer $(100 \mathrm{mmol} / \mathrm{L}$ $\mathrm{Na}_{2}$-EDTA, $10 \mathrm{mmol} / \mathrm{L}$ Tris, $2.5 \mathrm{~mol} / \mathrm{L} \mathrm{NaCl}, \mathrm{pH}$ 11,

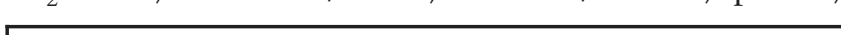

This work is licensed under a Creative Commons Attribution-NonCommercial-NoDerivatives 4.0 International License. International ficense.
ded from Bioscientifica.com at 04/26/2023 01:50:43PM 
$4 \mathrm{mmol} / \mathrm{L}$ dithiothreitol, and 2\% Triton X-100), was added and kept at $4^{\circ} \mathrm{C}$ for $2 \mathrm{~h}$. The slides were washed twice in distilled water for $5 \mathrm{~min}$ to remove the excess salts. Then, they were immersed in alkaline electrophoresis solution (300 mmol/L NaOH, $1 \mathrm{mmol} / \mathrm{L} \mathrm{Na}_{2}$-EDTA $\mathrm{pH}>13$ ) at 1.5 $\mathrm{V} / \mathrm{cm},<200 \mathrm{~mA}$, for $20 \mathrm{~min}$. The slides were then washed twice in TBE buffer for $5 \mathrm{~min}$, and after that, fixed in 70 and $100 \%$ ethanol. After drying, the slides were stained with Syber Green in TBE $(1 \mu \mathrm{L}$ Syber Green in $10 \mathrm{~mL}$ of TBE) for $40 \mathrm{~min}$. The slides were washed with TBE to remove background staining. They were evaluated using an Olympus BX51 epifluorescence microscope equipped with a rhodamine/TRITC filter under 400× magnification. 100 sperm (50 sperm per slide) were scored according to the intensity of DNA damage observed by the Comet tail and nuclear intensity. The sperm were classified as Comet grade I (observed nucleus and no DNA migration), Comet grade II (observed nucleus and little DNA migration), Comet grade III (observed nucleus and intense DNA migration), and Comet grade IV (no observed nucleus and intense DNA migration) (Fig. 1D). The percentage of each grade of sample was then calculated.

\section{Evaluation of epididymal function}

The epididymal function was evaluated through neutral alpha-glucosidase (NAG) activity according to WHO (2010) (11742027001 - Roche). Briefly, $100 \mu \mathrm{L}$ of seminal plasma (centrifuged at $1000 \boldsymbol{g}$ and pre-frozen) was thawed, and the samples were tested in accordance with the manufacturer's recommendations. The absorbance was measured by spectrophotometry (ELx 800 Absorbance Microplate Reader, Biotek) at a wavelength of $405 \mathrm{~nm}$. The NAG activity in the samples was determined as $\mathrm{mU}$ per ejaculate.

\section{Statistical analysis}

For this study, seminal samples from 65 men was obtained; however, 15 men were excluded due to semen analysis alterations (see item results). Thus, two seminal samples ( 1 and 4 days of EA) from 50 men were used for statistical analysis. For this purpose, PASW (SPSS) 18.0 software for Windows was used. Initially, the Kolmogorov-Smirnov test was used to verify the normality of data distribution. Then, for variables with normal distribution, a Student's $t$-test for paired samples was performed. For variables without normal distribution, the non-parametric Wilcoxon signed-rank test was performed. An alpha of $5 \%$ was adopted.

\section{Results}

Even though the participants spontaneously contacted and agreed to participate, $30 \%$ of the men who agreed to participate in the study did not attend the scheduled laboratory collection.

The semen samples from 65 men after 1 and 4 days of EA were analyzed. However, 15 men were excluded due to semen analysis abnormalities in both samples: 7 men showed samples with seminal volume $<1 \mathrm{~mL}, 4$ men showed samples with sperm concentration $<5 \times 10^{6} / \mathrm{mL}$, and 4 men were leukocytospermic.

The conventional semen analysis results are shown in Table 2. Liquefaction time showed no difference between the two analyzed samples ( 1 and 4 days after EA). As expected, the samples collected after 4 days of EA showed an increase in volume and total sperm number compared with samples collected after 1 day of EA $(P<0.001$ and $P<0.001$, respectively; Table 2). On the other hand, the samples collected after 1 day of EA showed better motility compared with samples collected after 4 days of EA (sperm total motility, $P=0.026$; progressive motility, $P=0.025$; and immotile, $P=0.041$; Table 2 ), as well as a higher $\mathrm{pH}$ value $(P<0.001$; Table 2$)$.

\section{Oxidative activity}

The oxidative activity results are shown in Table 3 . The samples collected after 1 day of EA showed a decrease in intracellular oxidative activity and seminal plasma oxidative activity compared with samples collected after 4 days of EA ( $P=0.006$ and $P=0.001$, respectively; Table 3$)$.

\section{Acrosome integrity, mitochondrial activity, nuclear DNA integrity}

The samples collected after 1 day of EA showed better acrosome integrity $(P<0.001$; Table 4$)$, mitochondrial activity (increased DAB class I, $P=0.041$ and decreased DAB class IV, $P<0.001$; Table 4 ), and nuclear DNA integrity (increased Comet grade I, $P<0.001$; decreased Comet grade II, $P=0.005$, and Comet grade IV, $P=0.008$; Table 4) compared with samples collected after 4 days of EA.

\section{Epididymal function}

Epididymal function showed no difference between the two analyzed samples ( 1 and 4 days of EA; Table 4 ).

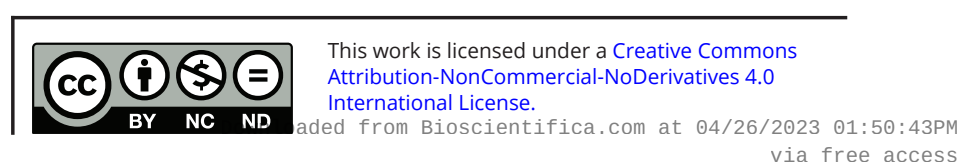


Table 2 Conventional semen analysis results from men after 1 and 4 days of ejaculatory abstinence. Data for liquefaction time, volume and motility data were compared using a Student's $t$-test for paired samples and are presented as mean \pm s.D. (95\% Cl) whilst $\mathrm{pH}$, sperm concentration, sperm total number, sperm normal morphology, rounds cell concentration and neutrophil concentration were compared using a Wilcoxon signed-ranks test and are presented as median; interquartile range (IQR: first and third quartile (Q1-Q3)).

\begin{tabular}{lcc} 
& & $\mathbf{1}$ day $(n=50)$ \\
\cline { 1 - 1 } Liquefaction time $(\mathrm{min})$ & $29.1 \pm 12.5(25.6 ; 32.5)$ \\
Volume $(\mathrm{mL})$ & $2.8 \pm 1.2(2.5 ; 3.2)$ \\
Sperm total motility** & $60.5 \pm 12.3(57.1 ; 64.0)$ \\
Progressive motility $(\%)$ & $55.1 \pm 12.3(51.7 ; 58.5)$ \\
Non-progressive motility $(\%)$ & $5.4 \pm 2.8(4.6 ; 6.2)$ \\
Immotile $(\%)$ & $39.5 \pm 12.3(36.0 ; 42.9)$ \\
pH & $8.2 ; 0.5(8.0-8.5)$ \\
Sperm concentration $\left(\times 10^{*} / \mathrm{mL}\right)$ & $53.4 ; 57.3(20.4-77.7)$ \\
Sperm total number $\left(\times 10^{\circ} / \mathrm{ejaculate}\right)$ & $120.1 ; 168.0(39.7-207.7)$ \\
Sperm normal morphology $(\%)$ & $6.0 ; 2.0(5.0-7.0)$ \\
Round cell concentration $(\times 106 / \mathrm{mL})$ & $0.8 ; 1.0(0.5-1.4)$ \\
Neutrophil concentration $\left(\times 10^{6} / \mathrm{mL}\right)$ & $0.0 ; 0.1(0.0-0.1)$ \\
\hline
\end{tabular}

\begin{tabular}{c}
\hline $\mathbf{4}$ days $(n=50)$ \\
\hline $29.5 \pm 12.2(26.1 ; 32.9)$ \\
$3.7 \pm 1.6(3.2 ; 4.1)$ \\
$57.5 \pm 11.9(54.2 ; 60.8)$ \\
$52.0 \pm 12.1(48.6 ; 55.3)$ \\
$5.5 \pm 2.2(4.9 ; 6.1)$ \\
$42.3 \pm 12.1(38.9 ; 45.6)$ \\
$8.0 ; 0.1(8.0-8.1)$ \\
$45.2 ; 76.5(19.6-96.1)$ \\
$147.5 ; 240.2(74.6-314.8)$ \\
$5.0 ; 3.0(4.0-7.0)$ \\
$0.7 ; 1.0(0.5-1.5)$ \\
$0.0 ; 0.1(0.0-0.1)$ \\
\hline
\end{tabular}

\begin{tabular}{c}
\hline $\boldsymbol{P}$ value \\
\hline 0.866 \\
$<0.001 *$ \\
$0.026 *$ \\
$0.025^{*}$ \\
0.781 \\
$0.041 *$ \\
$<0.001 *$ \\
0.337 \\
$<0.001 *$ \\
0.395 \\
0.273 \\
0.503
\end{tabular}

$* * \%$ progressive + non-progressive; $* P<0.05$

\section{Discussion}

The relationship between sperm and oxidative stress has been studied for a long time. The purpose of this study was to determine which qualitative semen characteristics may be affected by the action of oxidative stress. Hence, two EA periods were considered: 1 and 4 days.

When only the outcomes of the conventional semen analysis were analyzed, the samples collected after 4 days of EA showed an increase in volume and total sperm number compared with samples collected after 1 day of EA. These findings are consistent with other studies (De Jonge et al. 2004, Levitas et al. 2005, Lehavi et al. 2014, Agarwal et al. 2016, Mayorga-Torres et al. 2016, Alipour et al. 2017, Hanson et al. 2018). On the other hand, sperm motility of samples collected after 1 day of EA was better compared with samples collected after 4 days of EA, as previously observed (Mayorga-Torres et al. 2016, Alipour et al. 2017).

According to Iwasaki \& Gagnon (1992), there is a correlation between a low percentage of motile sperm and high oxidative stress. This study also reports similar results in samples collected after 4 days of EA. When ROS are present in physiological quantities, they contribute to the processes of maturation, hyperactivation, capacitation, the acrosome reaction, attachment of spermatozoa to the zona pellucida, and fusion of spermatozoon to the oolemma. However, an imbalance between ROS and antioxidants generates oxidative stress and leads to lipid peroxidation, a reaction with polyunsaturated fatty acids of the plasma and mitochondrial membranes of the sperm (Aitken et al. 2012). This phenomenon produces a product called malondialdehyde, a stable and mutagenic substance that can covalently bind to DNA nucleotides, peptides, and proteins and change their molecular functions. Thus, it can damage the acrosome, impair mitochondrial activity, and alter nuclear DNA integrity (de Lamirande et al. 1997). Consistently, samples collected after 4 days of EA an increase in oxidative stress determined by seminal plasma oxidative activity (TBARS) and intracellular oxidative activity (superoxide anion) was observed.

Table 3 Oxidative stress analysis results from men after 1 and 4 days of ejaculatory abstinence. Intracellular oxidative activity was compared using a Student's t-test for paired samples and data is presented as mean \pm S.D. (95\% Cl). Seminal plasma oxidative activity was compared using a Wilcoxon signed-ranks test and data is presented as median; interquartile range (IQR; (Q1-Q3)).

\begin{tabular}{|c|c|c|c|}
\hline & 1 day $(n=50)$ & 4 days $(n=50)$ & $P$ value \\
\hline Intracellular oxidative activity ${ }^{\dagger}$ & $15.3 \pm 19.0(10.0 ; 20.5)$ & $18.6 \pm 17.5(13.8 ; 23.5)$ & $0.006 *$ \\
\hline Seminal plasma oxidative activity ${ }^{\ddagger}$ & $6.9 ; 3.2(5.4-8.5)$ & $7.6 ; 2.5(6.7-9.1)$ & $0.001 *$ \\
\hline
\end{tabular}

${ }^{\star} P<0.05$; †superoxide anion - \% DHE-positive; ${ }^{\ddagger}$ MDA/lipid peroxidation - TBARS nmol/mL.

$\mathrm{DHE}$, dihydroethidium probe; MDA, malondialdehyde; TBARS, thiobarbituric acid reactive substances.

https://raf.bioscientifica.com

https://doi.org/10.1530/RAF-20-0018 (c) 2020 The authors Published by Bioscientifica Ltd

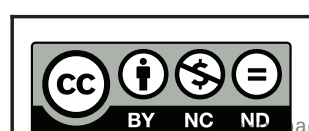

This work is licensed under a Creative Commons Attribution-NonCommercial-NoDerivatives 4.0 International License.
ded from Bioscientifica.com at 04/26/2023 01:50:43PM via free access 
Table 4 Sperm function and epididymal function analysis results from men after 1 and 4 days of ejaculatory abstinence. Acrosome integrity, mitochondrial activity and DNA integrity were compared using a Wilcoxon signed-ranks test and data is presented as median; interquartile range (IQR (Q1-Q3)). Epididymal function was compared using a Student's $t$-test for paired samples and data is presented as mean \pm S.D. $(95 \% \mathrm{CI})$.

\begin{tabular}{|c|c|c|c|}
\hline & 1 day $(n=50)$ & 4 days $(n=50)$ & $P$ value \\
\hline Acrosome integrity $^{\dagger}$ & $88.0 ; 8.9$ (83.0-91.9) & $83.7 ; 7.4(80.1-87.5)$ & $<0.001 *$ \\
\hline \multicolumn{4}{|l|}{ Mitochondrial activity (\%) } \\
\hline DAB class I & $7.2 ; 16.1(2.2-18.4)$ & $5.2 ; 10.3(3.1-13.4)$ & 0.041 * \\
\hline DAB class II & $67.7 ; 19.9$ (56.0-75.9) & $65.5 ; 20.9$ (54.9-75.7) & 0.429 \\
\hline DAB class III & $16.7 ; 13.1(11.2-24.4)$ & $16.5 ; 17.7(11.2-29.0)$ & 0.429 \\
\hline DAB class IV & $2.2 ; 3.4(1.0-4.4)$ & $3.5 ; 3.5(2.0-5.5)$ & $<0.001 *$ \\
\hline \multicolumn{4}{|l|}{ DNA integrity (\%) } \\
\hline Comet grade I & $43.5 ; 28.0(24.7-52.7)$ & $29.5 ; 24.2(16.5-40.7)$ & $<0.001 *$ \\
\hline Comet grade II & $44.5 ; 27.1(32.0-59.1)$ & $60.0 ; 29.0$ (40.0-69.0) & $0.005^{*}$ \\
\hline Comet grade III & $8.0 ; 6.7(4.2-11.0)$ & $8.5 ; 7.0(5.0-12.0)$ & 0.484 \\
\hline Comet grade IV & $3.0 ; 5.7(1.0-6.7)$ & $5.0 ; 5.9(3.0-8.9)$ & $0.008 *$ \\
\hline Epididymal function ${ }^{\ddagger}$ & $30.8 \pm 17.0(22.8 ; 38.9)$ & $37.6 \pm 18.4(28.9 ; 46.3)$ & 0.055 \\
\hline
\end{tabular}

t\% intact acrosome; *NAG mU/ejaculate; $* P<0.05$.

DAB, 3,3-diaminobenzidine chromogen; NAG, neutral alpha-glucosidase.

An increase in ROS levels in seminal plasma leads to oxidative stress. ROS can be produced by two cells: leukocytes and sperm. In the present study, we excluded men with leukocytospermia; in this context, sperm are the largest producers of ROS. Electrons are generated in sperm by the electron transport chain (mitochondrial origin) or NAD phosphate (NADPH) oxidase (plasma membrane; Aitken 2002, De Iuliis et al. 2006). In this study, intracellular oxidative activity was analyzed by the presence of superoxide anion, a free radical generated by the addition of an electron to an oxygen molecule. For samples collected after 4 days of EA, there was an increase in intracellular oxidative activity (superoxide anion) and a decrease in mitochondrial activity. These results agree with the findings described above, with regard to the non-mitochondrial origin. In a previous study carried out by our group (Adami et al. 2018), the same correlation in sperm incubated with an oxidative stress inductor was shown. Specifically, an increase in intracellular oxidative activity (superoxide anion) and a decrease in mitochondrial activity was found. According to Said et al. (2004), high levels of intracellular oxidative activity (superoxide anion) often occur in seminal samples with a low sperm concentration, as well as sperm that have low motility and exhibit abnormal morphology. Although morphology and concentration did not differ in this study, the motility in samples collected after 4 days of EA showed similar results, namely a high level of intracellular oxidative activity (superoxide anion) and low motility.

Some studies have shown that abnormal sperm are more susceptible to oxidative stress damage. Abnormal sperm may be generated by the failure of compaction of the chromatin (protamine), failure of apoptosis, and failure of phagocytosis by Sertoli cells (Sakkas et al. 2002). These sperm may suffer from the actions of oxidative stress from the intracellular or extracellular environment or both. Intracellular oxidative stress is generated by the sperm through electrons from mitochondria and/or NADPH oxidase. Extracellular oxidative stress from the seminal plasma can act on sperm. The combination of intracellular and extracellular stress can lead to acrosomal, mitochondrial, and DNA damage. In the samples collected after 4 days of EA, the action of oxidative stress on the acrosome was observed. In this case, oxidative stress may have acted on sperm plasma membrane glycoproteins, which may have been disrupted by lipid peroxidation.

Oxidative stress can also act on the genetic material of sperm and lead to nuclear DNA fragmentation. The samples collected after 4 days of EA showed reduced DNA integrity compared with samples collected after 1 day of EA, as observed by Agarwal et al. (2016) and Borges et al. (2019), who compared samples collected after 4 days of EA with a period of less than 4 days of EA. The majority of sperm nuclear DNA is compacted by protamines; the rest is compacted by persistent histones. According to Noblanc et al. (2013), the regions of DNA compacted by histones are more vulnerable to damage. Further, in mice, persistent histones are responsible for compaction of about $1-2 \%$ of the sperm DNA. In humans, persistent histones are responsible for compaction of approximately $15 \%$ of the sperm DNA; they require greater attention due to their fragility. This vulnerability can be worsened by a 
long-sperm storage period in the epididymal tail, which can increase oxidative stress, as observed in samples collected after 4 days of EA compared to samples collected after 1 day of EA.

Another factor that could render sperm more susceptible is epididymal dysfunction, whether the sperm remain in the epididymis for a long time, the period of sperm transit (through the epididymal head, body, and tail), and the period of EA. NAG activity has been used to measure epididymal function (WHO 2010). NAG is produced and secreted by epididymal epithelial cells (Guerin et al. 1986); therefore, it is considered the most sensitive epididymal marker (more so than L-carnitine and glycerophosphocholine). NAG activity is significantly different between fertile and infertile men (Cooper 1996, Vivas-Acevedo et al. 2014, Goss et al. 2019); however, in this study, differences in the activity in samples collected after 1 and 4 days of EA was not observed.

Besides the epididymis and testis, the prostate and seminal vesicle are essential organs for fertility. Furthermore, the secretions of these last two tissues can directly interfere with the volume and $\mathrm{pH}$ of the seminal plasma (WHO 2010). As stated earlier, the samples collected after 1 day of EA were more alkaline compared to samples after 4 days of EA. According to the WHO guidelines, in an acidic environment, sperm are immobile, while in an alkaline environment, sperm motility increases. However, both analyzed periods showed optimum $\mathrm{pH}$ (7-8.5) for sperm migration and survival (WHO 2010). Two accessory organs are responsible for the majority of seminal plasma secretions: the prostate and seminal vesicle. Goss et al. (2019), who used prostate (citric acid) and seminal vesicle (fructose) markers in seminal samples, observed a reduction in these markers in samples collected after $4 \mathrm{~h}$ of EA when compared with samples collected after 4 days of EA. The accessory gland secretions decreased with the reduced EA period, thus diminishing the alkaline secretion of the seminal vesicle and the acid secretion of the prostate, causing only a partial imbalance of the final $\mathrm{pH}$, as observed in our study.

Besides volume and $\mathrm{pH}$, the prostate and seminal vesicle can interfere in the oxidative stress of seminal plasma. The TBARS assays measured the oxidative stress of seminal plasma. Samples collected after 4 days of EA showed an increase in oxidative stress compared to samples collected after 1 day of EA.

In addition to oxidative stress, other factors may interfere with male fertility, such as endocrine disruptors (Lymperi \& Giwercman 2018). These molecules are increasingly present in our environment: air, water, soil, food, and industrial products, among others. Therefore, they are difficult to eliminate from contact with humans. However, in this study, the two seminal samples (1 day and 4 days of EA) were collected in the same week to reduce the bias of the endocrine disruptors on seminal samples.

Our study brought additional information placed alongside other studies and of medical facts can facilitate clinical decision-making on which procedure should be performed.

\section{Conclusion}

In conclusion, our study verified that the increase in oxidative stress on samples collected after 4 days of EA led to a decrease in acrosome integrity, mitochondrial activity, and nuclear DNA integrity compared with samples collected after 1 day of EA. Therefore, a longsperm storage period in the epididymal tail (4 days of EA), beyond the time required for sperm transit, allows ROS to act for a longer period on sperm, which affects the sperm quality. These results may be considered to obtain better results in assisted reproduction techniques in order to improve both fertilization and implantation rates.

\section{Supplementary materials}

This is linked to the online version of the paper at https://doi.org/10.1530/ RAF-20-0018.

\section{Declaration of interest}

The authors declare that there is no conflict of interest that could be perceived as prejudicing the impartiality of the research reported.

\section{Funding}

The funding for this project was received from the Coordenação de Aperfeiçoamento de Pessoal de Nível Superior (CAPES) as a scholarship for PhD Okada.

\section{Ethical approval}

This study received institutional review board approval from the São Paulo Federal University Research Ethics Committee (CAAE: 19519913.8.0000.5505).

\section{Author contribution statement}

F K O concept and design of the research, carrying out the experimental work, data analysis and interpretation, writing the article and revision of

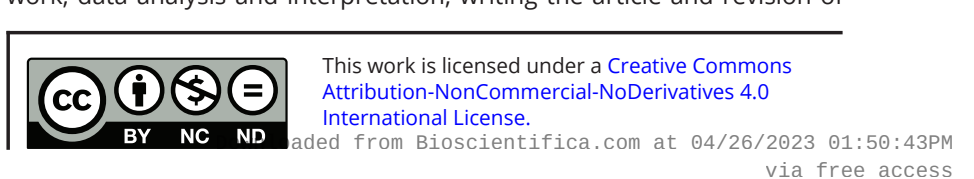


the manuscript. R R A carrying out the experimental work, data analysis and interpretation, revision of the manuscript. D M S concept and design of the research, data analysis and interpretation, writing of the article and revision of the manuscript.

\section{References}

Acuña RG, Schwarze MJE, Villa VS \& Pommer TR 2013 Influencia de la abstinencia eyaculatoria en las características del espermiograma: revisión sistemática. Revista Chilena de Obstetricia y Ginecología $\mathbf{7 8}$ 290-292. (https://doi.org/10.4067/S0717-75262013000400007)

Adami LNG, Belardin LB, Lima BT, Jeremias JT, Antoniassi MP, Okada FK \& Bertolla RP 2018 Effect of in vitro vitamin E (alphatocopherol) supplementation in human spermatozoon submitted to oxidative stress. Andrologia 50 e12959. (https://doi.org/10.1111/ and.12959)

Agarwal A, Gupta S, Du Plessis S, Sharma R, Esteves SC, Cirenza C, Eliwa J, Al-Najjar W, Kumaresan D, Haroun N et al. 2016 Abstinence time and its impact on basic and advanced semen parameters. Urology 94 102-110. (https://doi.org/10.1016/j. urology.2016.03.059)

Aitken RJ 2002 Changes in spermatozoa during epididymal transit. Active oxygen in spermatozoa during epididymal transit. In The Epididymis: From Molecules to Clinical Practice, pp. 435-445. Eds B Robaire \& B Hinton. New York: Kluwer Academic/Plenum Publishers

Aitken RJ, Jones KT \& Robertson SA 2012 Reactive oxygen species and sperm function - in sickness and in health. Journal of Andrology 33 1096-1106. (https://doi.org/10.2164/jandrol.112.016535)

Alipour H, Van Der Horst G, Christiansen OB, Dardmeh F, Jørgensen N, Nielsen HI \& Hnida C 2017 Improved sperm kinematics in semen samples collected after $2 \mathrm{~h}$ versus 4-7 days of ejaculation abstinence. Human Reproduction 32 1364-1372. (https:// doi.org/10.1093/humrep/dex101)

Ayad BM, der Horst GV \& Plessis SSD 2018 Revisiting the relationship between the ejaculatory abstinence period and semen characteristics. International Journal of Fertility and Sterility $\mathbf{1 1}$ 238-246. (https://doi.org/10.22074/ijfs.2018.5192)

Björndahl L, Barratt CLR, Mortimer D \& Jouannet P 2016 'How to count sperm properly': checklist for acceptability of studies based on human semen analysis. Human Reproduction 31 227-232. (https:// doi.org/10.1093/humrep/dev305)

Blumer CG, Fariello RM, Restelli AE, Spaine DM, Bertolla RP \& Cedenho AP 2008 Sperm nuclear DNA fragmentation and mitochondrial activity in men with varicocele. Fertility and Sterility 90 1716-1722. (https://doi.org/10.1016/j.fertnstert.2007.09.007)

Blumer CG, Restelli AE, Giudice PTD, Soler TB, Fraietta R, Nichi M, Bertolla RP \& Cedenho AP 2012 Effect of varicocele on sperm function and semen oxidative stress. BJU International 109 259-265. (https://doi.org/10.1111/j.1464-410X.2011.10240.x)

Borges E, Braga DPAF, Zanetti BF, Iaconelli A \& Setti AS 2019 Revisiting the impact of ejaculatory abstinence on semen quality and intracytoplasmic sperm injection outcomes. Andrology 7 213-219. (https://doi.org/10.1111/andr.12572)

Carlsen E, Petersen JH, Andersson AM \& Skakkebaek NE 2004 Effects of ejaculatory frequency and season on variations in semen quality. Fertility and Sterility 82 358-366. (https://doi.org/10.1016/j. fertnstert.2004.01.039)

Cooper TG 1996 Epididymis and sperm function. Andrologia 28 (Supplement 1) 57-59. (https://doi.org/10.1111/j.1439-0272.1996. tb02758.x)

De Iuliis GN, Wingate JK, Koppers AJ, McLaughlin EA \& Aitken RJ 2006 Definitive evidence for the nonmitochondrial production of superoxide anion by human spermatozoa. Journal of
Clinical Endocrinology and Metabolism 91 1968-1975. (https://doi. org/10.1210/jc.2005-2711)

De Jonge C, LaFromboise M, Bosmans E, Ombelet W, Cox A \& Nijs M 2004 Influence of the abstinence period on human sperm quality. Fertility and Sterility 82 57-65. (https://doi.org/10.1016/j. fertnstert.2004.03.014)

de Lamirande E, Jiang H, Zini A, Kodama H \& Gagnon C 1997 Reactive oxygen species and sperm physiology. Reviews of Reproduction 2 48-54. (https://doi.org/10.1530/ror.0.0020048)

Goss D, Ayad B, van der Horst G, Skosana B \& du Plessis SS 2019 Improved sperm motility after $4 \mathrm{~h}$ of ejaculatory abstinence: role of accessory sex gland secretions. Reproduction, Fertility, and Development 31 1009-1016. (https://doi.org/10.1071/RD18135)

Guerin JF, Ali HB, Rollet J, Souchier C \& Czyba JC 1986 Alphaglucosidase as a specific epididymal enzyme marker. Its validity for the etiologic diagnosis of azoospermia. Journal of Andrology 7 156-162. (https://doi.org/10.1002/j.1939-4640.1986.tb00901.x)

Hanson BM, Aston KI, Jenkins TG, Carrell DT \& Hotaling JM 2018 The impact of ejaculatory abstinence on semen analysis parameters: a systematic review. Journal of Assisted Reproduction and Genetics 35 213-220. (https://doi.org/10.1007/s10815-017-1086-0)

Hrudka F 1987 Cytochemical and ultracytochemical demonstration of cytochrome c oxidase in spermatozoa and dynamics of its changes accompanying ageing or induced by stress. International Journal of Andrology 10 809-828. (https://doi.org/10.1111/j.1365-2605.1987. tb00385.x)

Intasqui P, Antoniassi MP, Camargo M, Nichi M, Carvalho VM, Cardozo KHM, Zylbersztejn DS \& Bertolla RP 2015 Differences in the seminal plasma proteome are associated with oxidative stress levels in men with normal semen parameters. Fertility and Sterility 104 292-301. (https://doi.org/10.1016/j.fertnstert.2015.04.037)

Intasqui P, Camargo M, Antoniassi MP, Cedenho AP, Carvalho VM, Cardozo KHM, Zylbersztejn DS \& Bertolla RP 2016 Association between the seminal plasma proteome and sperm functional traits. Fertility and Sterility 105 617-628. (https://doi. org/10.1016/j.fertnstert.2015.11.005)

Iwasaki A \& Gagnon C 1992 Formation of reactive oxygen species in spermatozoa of infertile patients. Fertility and Sterility $\mathbf{5 7} 409-416$. (https://doi.org/10.1016/s0015-0282(16)54855-9)

Lehavi O, Botchan A, Paz G, Yogev L, Kleiman SE, Yavetz H \& Hauser R 2014 Twenty-four hours abstinence and the quality of sperm parameters. Andrologia 46 692-697. (https://doi.org/10.1111/and.12137)

Levitas E, Lunenfeld E, Weiss N, Friger M, Har-Vardi I, Koifman A \& Potashnik G 2005 Relationship between the duration of sexual abstinence and semen quality: analysis of 9,489 semen samples. Fertility and Sterility 83 1680-1686. (https://doi. org/10.1016/j.fertnstert.2004.12.045)

Levitas E, Lunenfeld E, Weisz N, Friger M, Har-Vardi I \& Potashnik G 2006 Relationship between sexual abstinence duration and the acrosome index in teratozoospermic semen: analysis of 1800 semen samples. Andrologia 38 110-112. (https://doi.org/10.1111/ j.1439-0272.2006.00715.x)

Lymperi S \& Giwercman A 2018 Endocrine disruptors and testicular function. Metabolism: Clinical and Experimental 86 79-90. (https:// doi.org/10.1016/j.metabol.2018.03.022)

Mahfouz RZ, du Plessis SS, Aziz N, Sharma R, Sabanegh E \& Agarwal A 2010 Sperm viability, apoptosis, and intracellular reactive oxygen species levels in human spermatozoa before and after induction of oxidative stress. Fertility and Sterility 93 814-821. (https://doi.org/10.1016/j.fertnstert.2008.10.068)

Marshburn PB, Giddings A, Causby S, Matthews ML, Usadi RS, Steuerwald N \& Hurst BS 2014 Influence of ejaculatory abstinence on seminal total antioxidant capacity and sperm membrane lipid peroxidation. Fertility and Sterility 102 705-710. (https://doi.org/10.1016/j.fertnstert.2014.05.039) (c) 2020 The authors Published by Bioscientifica Ltd
This work is licensed under a Creative Commons Attribution-NonCommercial-NoDerivatives 4.0 International License. International License.
ded from Bioscientifica.com at 04/26/2023 01:50:43PM 
Mayorga-Torres BJM, Camargo M, Agarwal A, du Plessis SS, Cadavid ÁP \& Cardona Maya WD 2015 Influence of ejaculation frequency on seminal parameters. Reproductive Biology and Endocrinology 13 47. (https://doi.org/10.1186/s12958-015-0045-9)

Mayorga-Torres JM, Agarwal A, Roychoudhury S, Cadavid A \& Cardona-Maya WD 2016 Can a short term of repeated ejaculations affect seminal parameters? Journal of Reproduction and Infertility $\mathbf{1 7}$ 177-183.

Noblanc A, Damon-Soubeyrand C, Karrich B, Henry-Berger J, Cadet R, Saez F, Guiton R, Janny L, Pons-Rejraji H, Alvarez JG et al. 2013 DNA oxidative damage in mammalian spermatozoa: where and why is the male nucleus affected? Free Radical Biology and Medicine 65 719-723. (https://doi.org/10.1016/j.freeradbiomed.2013.07.044)

Ohkawa H, Ohishi N \& Yagi K 1979 Assay for lipid peroxides in animal tissues by thiobarbituric acid reaction. Analytical Biochemistry 95 351-358. (https://doi.org/10.1016/0003-2697(79)90738-3)

Pons I, Cercas R, Villas C, Braña C \& Fernández-Shaw S 2013 One abstinence day decreases sperm DNA fragmentation in $90 \%$ of selected patients. Journal of Assisted Reproduction and Genetics 30 1211-1218. (https://doi.org/10.1007/s10815-013-0089-8)

Rivaroli M 2009 Comparison between length of sexual abstinence and semen parameters in patients of an assisted reproduction center and a hospital from Porto Alegre. Research Series 13 28-32.

Said TM, Agarwal A, Sharma RK, Mascha E, Sikka SC \& Thomas AJ 2004 Human sperm superoxide anion generation and correlation with semen quality in patients with male infertility. Fertility and Sterility 82 871-877. (https://doi.org/10.1016/j. fertnstert.2004.02.132)

Sakkas D, Moffatt O, Manicardi GC, Mariethoz E, Tarozzi N \& Bizzaro D 2002 Nature of DNA damage in ejaculated human spermatozoa and the possible involvement of apoptosis. Biology of Reproduction 66 1061-1067. (https://doi.org/10.1095/ biolreprod66.4.1061)

Sánchez-Martín P, Sánchez-Martín F, González-Martínez M \& Gosálvez J 2013 Increased pregnancy after reduced male abstinence. Systems Biology in Reproductive Medicine 59 256-260. (https://doi.org/1 $0.3109 / 19396368.2013 .790919)$
Shen ZQ, Shi B, Wang TR, Jiao J, Shang XJ, Wu QJ, Zhou YM, Cao TF, Du Q, Wang XX et al. 2019 Characterization of the sperm proteome and reproductive outcomes with in vitro, fertilization after a reduction in male ejaculatory abstinence period. Molecular and Cellular Proteomics 18 S109-S117. (https://doi.org/10.1074/mcp. RA117.000541)

Sobreiro BP, Lucon AM, Pasqualotto FF, Hallak J, Athayde KS \& Arap S 2005 Semen analysis in fertile patients undergoing vasectomy: reference values and variations according to age, length of sexual abstinence, seasonality, smoking habits and caffeine intake. Sao Paulo Medical Journal 123 161-166. (https://doi.org/10.1590/ s1516-31802005000400002)

Uppangala S, Mathai SE, Salian SR, Kumar D, Singh VJ, D'Souza F, Kalthur G, Kamath A \& Adiga SK 2016 Sperm chromatin immaturity observed in short abstinence ejaculates affects DNA integrity and longevity in vitro. PLOS ONE 11 e0152942. (https://doi.org/10.1371/journal.pone.0152942)

Vivas-Acevedo G, Lozano-Hernández R \& Camejo MI 2014 Varicocele decreases epididymal neutral $\alpha$-glucosidase and is associated with alteration of nuclear DNA and plasma membrane in spermatozoa. BJU International 113 642-649. (https://doi. org/10.1111/bju.12523)

Welliver C, Benson AD, Frederick L, Leader B, Tirado E, Feustel P, Kontio J, McAsey M \& Köhler TS 2016 Analysis of semen parameters during 2 weeks of daily ejaculation: a first in humans study. Translational Andrology and Urology 5 749-755. (https://doi.org/10.21037/tau.2016.08.20)

World Health Organization 2010 WHO Laboratory Manual for the Examination and Processing of Human Semen, 5th ed. Geneva Switzerland: WHO Press.

Received in final form 2 September 2020

Accepted 11 September 2020

Accepted Manuscript published online 11 September 2020 https://raf.bioscientifica.com

https://doi.org/10.1530/RAF-20-0018 (c) 2020 The authors Published by Bioscientifica Ltd
This work is licensed under a Creative Commons Attribution-NonCommercial-NoDerivatives 4.0 International License. ded from Bioscientifica.com at 04/26/2023 01:50:43PM 